\title{
Novel Variations in MYO5B Presenting as Isolated Intrahepatic Cholestasis: Long-Term Outcome after Partial Internal Biliary Diversion
}

\author{
Bikrant Bihari Lal ${ }^{1}$ (D) $\cdot$ Vikrant Sood $^{1} \cdot$ Rajeev Khanna $^{1} \cdot$ Seema Alam $^{1}$ (D)
}

Received: 30 March 2021 / Accepted: 7 June 2021 / Published online: 22 July 2021

(c) Dr. K C Chaudhuri Foundation 2021

To the Editor: Mutations in myosin-5B (MYO5B) have recently been reported to present as isolated intrahepatic cholestasis without intestinal involvement; closely mimicking progressive familial intrahepatic cholestasis (PFIC) types 1 or 2 with similar age of onset, clinical features, presence of low gamma-glutamyl transferase (GGT) cholestasis, elevated serum bile acids (BA), and histological findings in children [1-3]. We report 2 siblings with novel compound heterozygous variations in $M Y O 5 B$ presenting as isolated cholestasis with excellent long-term response after partial internal biliary diversion (PIBD).

A 15 -y-old girl presented in 2015 with intractable pruritus since 9 mo of age refractory to medical therapy and growth failure. She had low-GGT cholestasis, elevated BA $(362 \mu \mathrm{mol} / \mathrm{L})$ and marked cholestasis with portal fibrosis on liver biopsy. Treating unit's provisional diagnosis was PFIC1/2. Genetic confirmation could not be done at that time. Child underwent PIBD (cholecysto-jejunocolonic anastomosis) with significant relief in pruritus and marked decrease in BA ( $362-->62 \mu \mathrm{mol} / \mathrm{L}$ ). Six years after PIBD, she remains pruritus free, with good catch-up growth (weight $z$ score: $-3.6-->-0.48$; height $z$ score: $-3.7-->-1.4$ ) and without fibrosis progression. Recently, her younger brother presented with infantile cholestasis at 7 mo of age and his genetic sequencing showed compound heterozygous variations in $M Y O 5 B$ gene. Following this, patient 1 also underwent genetic sequencing which revealed same compound heterozygous mutations in the MYO5B gene (chr18:47462664T>C; p.Tyr654Cys) and (chr18:47480695G $>$ C; p.His552Gln). Both the observed variations are novel mutations, lie in motor domain (myosin head) of MYO5B protein and are disease causing as per various in silico prediction tools.

Seema Alam

seema_alam@hotmail.com

1 Department of Pediatric Hepatology, Institute of Liver and Biliary Sciences, New Delhi 110070, India
$M Y O 5 B$ in combination with RAB 11 is involved in normal trafficking of $\mathrm{ABC}$ transporter proteins to the canalicular membrane [3, 4]. MYO5B mutations impair MYO5B/ $\mathrm{RAB} 11 \mathrm{~A}$ interaction leading to impaired BSEP transport to canalicular membrane causing decreased canalicular bile secretion $[3,4]$. Biliary diversion surgery dramatically improves pruritus, reduces serum bile acids, and improves growth in those refractory to medical therapy.

\section{Declarations}

Conflict of Interest None

\section{References}

1. Gonzales E, Taylor SA, Davit-Spraul A, et al. MYO5B mutations cause cholestasis with normal serum gamma-glutamyl transferase activity in children without microvillous inclusion disease. Hepatology. 2017;65:164-73.

2. Qiu YL, Gong JY, Feng JY, et al. Defects in myosin VB are associated with a spectrum of previously undiagnosed low $\gamma$-glutamyltransferase cholestasis. Hepatology. 2017;66:1708-9.

3. Girard M, Lacaille F, Verkarre V, et al. MYO5B and bile salt export pump contribute to cholestatic liver disorder in microvillous inclusion disease. Hepatology. 2014;60:301-10.

4. Overeem AW, Li Q, Qiu YL, et al. A molecular mechanism underlying genotype-specific intrahepatic cholestasis resulting from MYO5B mutations. Hepatology. 2020;72:213-29.

Publisher's Note Springer Nature remains neutral with regard to jurisdictional claims in published maps and institutional affiliations. 